ARTICLE

\title{
Septins promote dendrite and axon development by negatively regulating microtubule stability via HDAC6-mediated deacetylation
}

\author{
Natsumi Ageta-Ishihara1, Takaki Miyata², Chika Ohshima1', Masahiko Watanabe ${ }^{3}$, Yoshikatsu Sato ${ }^{4}$, \\ Yuki Hamamura', Tetsuya Higashiyama ${ }^{4}$, Ralph Mazitschek ${ }^{5}$, Haruhiko Bito ${ }^{6} \&$ Makoto Kinoshita ${ }^{1}$
}

Neurite growth requires two guanine nucleotide-binding protein polymers of tubulins and septins. However, whether and how those cytoskeletal systems are coordinated was unknown. Here we show that the acute knockdown or knockout of the pivotal septin subunit SEPT7 from cerebrocortical neurons impairs their interhemispheric and cerebrospinal axon projections and dendritogenesis in perinatal mice, when the microtubules are severely hyperacetylated. The resulting hyperstabilization and growth retardation of microtubules are demonstrated in vitro. The phenotypic similarity between SEPT7 depletion and the pharmacological inhibition of $\alpha$-tubulin deacetylase HDAC6 reveals that HDAC6 requires SEPT7 not for its enzymatic activity, but to associate with acetylated $\alpha$-tubulin. These and other findings indicate that septins provide a physical scaffold for HDAC6 to achieve efficient microtubule deacetylation, thereby negatively regulating microtubule stability to an optimal level for neuritogenesis. Our findings shed light on the mechanisms underlying the HDAC6-mediated coupling of the two ubiquitous cytoskeletal systems during neural development.

\footnotetext{
${ }^{1}$ Division of Biological Sciences, Nagoya University Graduate School of Science, Nagoya 464-8602, Japan. ${ }^{2}$ Department of Anatomy and Cell Biology, Nagoya University Graduate School of Medicine, Nagoya 466-8550, Japan. ${ }^{3}$ Department of Anatomy, Hokkaido University Graduate School of Medicine, Sapporo 060-8638, Japan. ${ }^{4}$ Institute of Transformative Bio-Molecules, Nagoya University, Nagoya 464-8602, Japan. ${ }^{5}$ Centre for Systems Biology, Massachusetts General Hospital and Harvard Medical School, Boston, Massachusetts 02114, USA. ${ }^{6}$ Department of Neurochemistry, Graduate School of Medicine, The University of Tokyo, Tokyo 113-0033, Japan. Correspondence and requests for materials should be addressed to M.K.

(email: kinoshita.makoto@c.mbox.nagoya-u.ac.jp).
} 
T he organization of the neural network depends on two distinct types of subcellular protrusions, dendrites and axons. Despite the diversity in morphology, composition and function, all neurites share a common structural backbone, the microtubule. The assembly and the balance between the dynamic instability and stability of microtubules are under multiplex control ${ }^{1}$. However, among the plethora of proteins that associate with microtubules, only a fraction of them has been assigned to physiological molecular pathways that control microtubules during neuritogenesis ${ }^{2}$.

Genetic studies have indicated that neuritogenesis in nematodes requires septins $s^{3,4}$, a family of heteropolymerizing guanosine tri-/di-phosphate-binding proteins whose mammalian homologues associate with microtubules in the brain $^{5}$. Although septin-null nematodes exhibit complex phenotypes, including neurite anomalies and uncoordinated locomotor defects ${ }^{6}$, a fundamental question remains as to whether the neurological defects are direct, or even cell autonomous, consequences due to the loss of septins.

Attempts with mouse reverse genetics so far have failed to provide informative findings as to whether and how mammalian septins (SEPT1-14) are required for neuritogenesis in vivo. The genetic disruption of one or two functionally redundant subunits within the septin family (that is, SEPT3, SEPT4, SEPT5, SEPT6, SEPT3 and SEPT5, or SEPT4 and SEPT6) gives no recognizable morphological anomaly in the nervous system, partly due to the adaptive upregulation of functionally similar subunits and/or other modes of compensation ${ }^{7-11}$. In contrast, embryos that lack the uniquely nonredundant subunit SEPT7 collapse by midgestation $^{12}$.

Mosaic analysis might offer a means to overcome the limitations of genetic approaches to characterize the redundant and multifunctional system of septins. We hypothesized that either local gene disruption or local acute depletion of a pivotal septin subunit from postmitotic, sprouting neurons might reveal a phenotype that is otherwise masked. A reasonable strategy would be to probe neuritogenesis in the developing brain via the local depletion of SEPT7 on the basis of the following evidence obtained in vitro: First, acute depletion of SEPT7 via RNA interference (RNAi) causes collateral destabilization and reduction of other subunits that constitute hetero-oligomers, which abolishes the entire septin system in mouse T lymphocytes ${ }^{13}$. Second, RNAi-mediated depletion of SEPT7 from rat hippocampal neurons in dissociation culture perturbs dendrite development, although the molecular mechanism and in vivo relevance of that phenomenon are unknown ${ }^{14,15}$.

The present study is designed for the parallel examination of in vivo and in vitro phenotypes of postmitotic cerebrocortical neurons after local and acute depletion of SEPT7 via RNAi or conditional gene disruption via the Cre-loxP recombination system. This approach reveals obvious neuritogenesis defects in vivo for the first time in vertebrates, and enables us to further investigate this mechanism in neurons dissociated in vitro, an experimental system which is more amenable to rigorous morphometry, molecular pharmacology, in situ antibody crosslinking assays and live imaging. Our combined approach allows us to uncover a novel septinnull phenotype in developing mouse brain, an unexpected mode of interaction between the microtubule and septin cytoskeletal systems, and a novel septin scaffold-based regulatory mechanism of HDAC6 as a deacetylase/adapter of $\alpha / \beta$ tubulins. We conclude that neuronal septins provide a scaffold for the efficient microtubule deacetylation by HDAC6, which optimizes the growth and stability of microtubule for neuritogenesis during pre- and postnatal development of mammalian brain.

\section{Results}

SEPT7 is required for neuritogenesis in the mouse brain. Previous in vitro studies have shown that primary neurons cultured from rat hippocampus and from the chick dorsal root ganglion require septins for dendrite development ${ }^{14,15}$ and axon branching ${ }^{16}$, respectively. To test the relationship and in vivo relevance of those findings and to explore the molecular mechanism(s) involved, we employed mouse cerebrocortical neurons, which enable both physiological analyses in vivo and rigorous morphometry and live imaging in vitro ${ }^{17-19}$. By immunoprecipitation and immunoblot analyses, we found that perinatal mouse cerebrocortical neurons express most of the septin subunits in vivo and in vitro as do rat hippocampal neurons ${ }^{15}$, and that SEPT5, SEPT6, SEPT7 and SEPT11 constitute the major septin species that form hetero-oligomers (Supplementary Fig. S1). To perturb the septin system, the pivotal subunit SEPT7 was depleted by expressing two distinct shRNAs against Sept7, when GFP was co-expressed as a marker of transfection and cell volume for morphometry ${ }^{20}$. The efficiency of SEPT7 depletion was $\sim 80 \%$ determined by immunofluorescence of GFP-positive cerebrocortical neurons electroporated and cultured in vitro (Fig. 1a) and by immunoblot of FACS-fractionated GFP-positive cerebrocortical neurons that had been electroporated in utero (Fig. 1b and Supplementary Fig. S2).

To test whether SEPT7 is required for neuritogenesis in a physiological context, we first focused on the perinatal development of pyramidal neurons in layer II/III of the primary and secondary somatosensory cortex (S1/S2). Each neuron emanates several dendrites apically and an axon basally to deeper layers, and the axon traverses the corpus callosum and projects towards layers II/III/V of the contralateral S1/S2. We injected two different shSept7/GFP plasmids with distinct target sequences (\#1 and \#2) into the left lateral ventricle beneath the left S1/S2 of E15 embryos in utero, and conducted electroporation targeting pyramidal neuron precursors destined to undergo radial migration to layer II/III. The migration, dendrite growth and interhemispheric axon projection of pyramidal neurons are known to complete by P15, when we conducted morphological analyses with coronal brain sections (Fig. 1c). We confirmed that local depletion of SEPT7 by either of those short hairpin RNAs (shRNAs) did not affect either the survival of neurons or their radial migration toward layer II/III (Supplementary Fig. S3). However, morphometry of GFP-positive pyramidal neurons in layer II/III of ipsilateral S1/S2 demonstrated statistically significant reductions in the total length and complexity (that is, branch tip number) of dendrites projecting locally from SEPT7-depleted neurons as compared with the control (Fig. 1d, left).

As morphometry was not applicable for individual axons in bundles, we measured the fluorescence intensity of the three neuronal segments; dendrites/somata at the ipsilateral S1/S2 layer II/III, the axon bundle at the corpus callosum and axon terminals at the contralateral S1/S2 layer II/III/V (Fig. 1c). The ratio of fluorescence intensity at the corpus callosum over that at dendrites/somata was comparable with or without SEPT7 depletion (Fig. 1e, top), indicating that depletion of SEPT7 does not significantly affect the axon projection up to the corpus callosum. In contrast, the ratio of fluorescence intensities and the branch numbers at the axon terminals over the fluorescence intensity at the corpus callosum was significantly reduced by depletion of SEPT7 (Fig. 1f, left), indicating defective extension and branching at the distal parts of axons. A follow-up observation at P30 corroborated that none of the morphological abnormality was corrected by weaning (Supplementary Fig. S4), indicating that perinatal depletion of SEPT7 causes irreversible defects in dendritogenesis and axonogenesis. Further, 

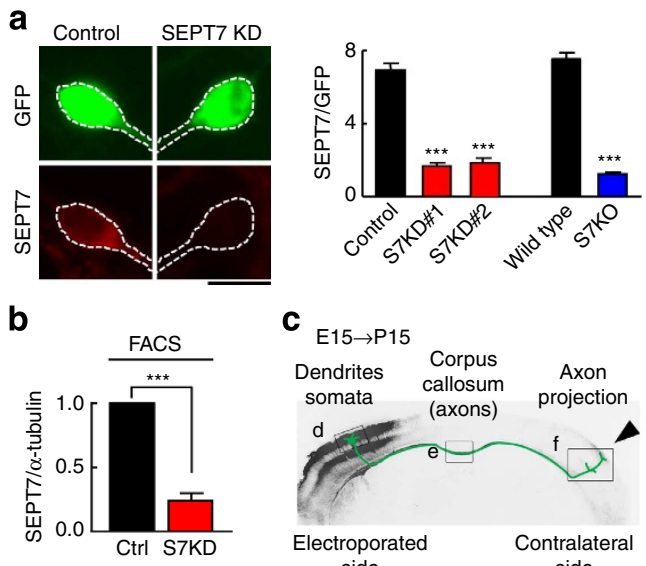

C

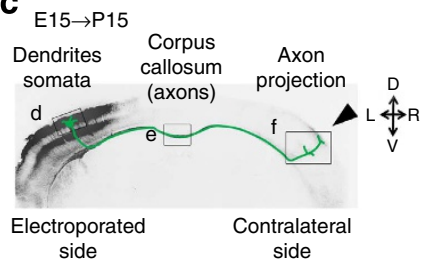
side d
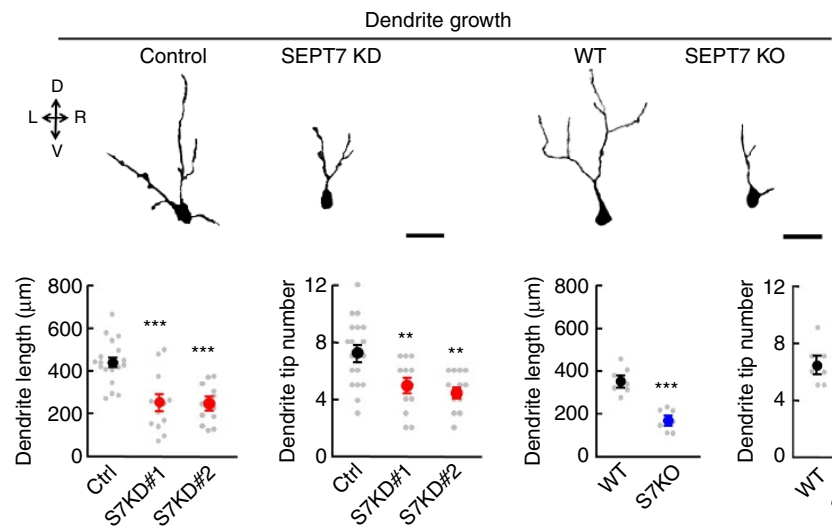
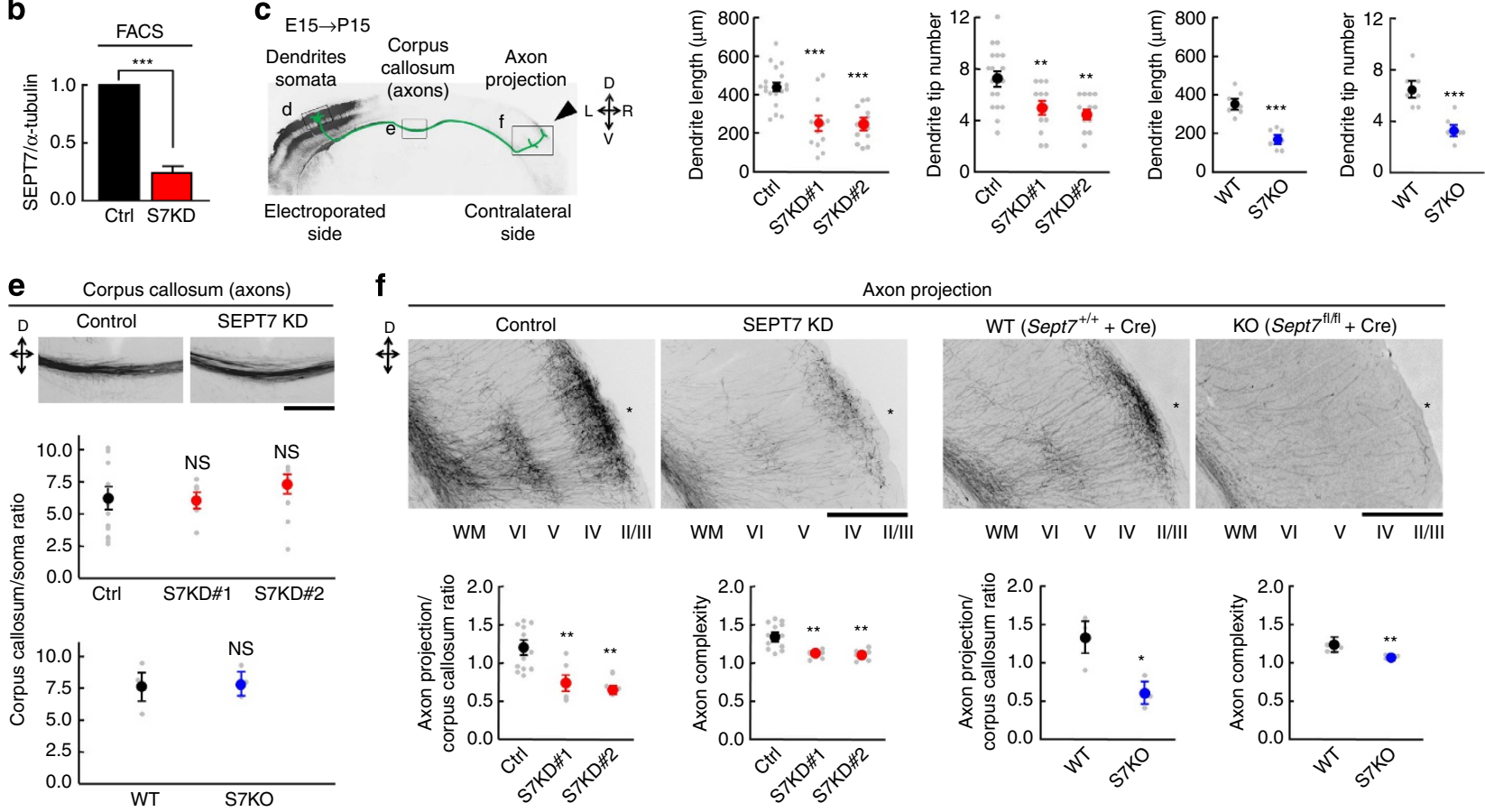

f
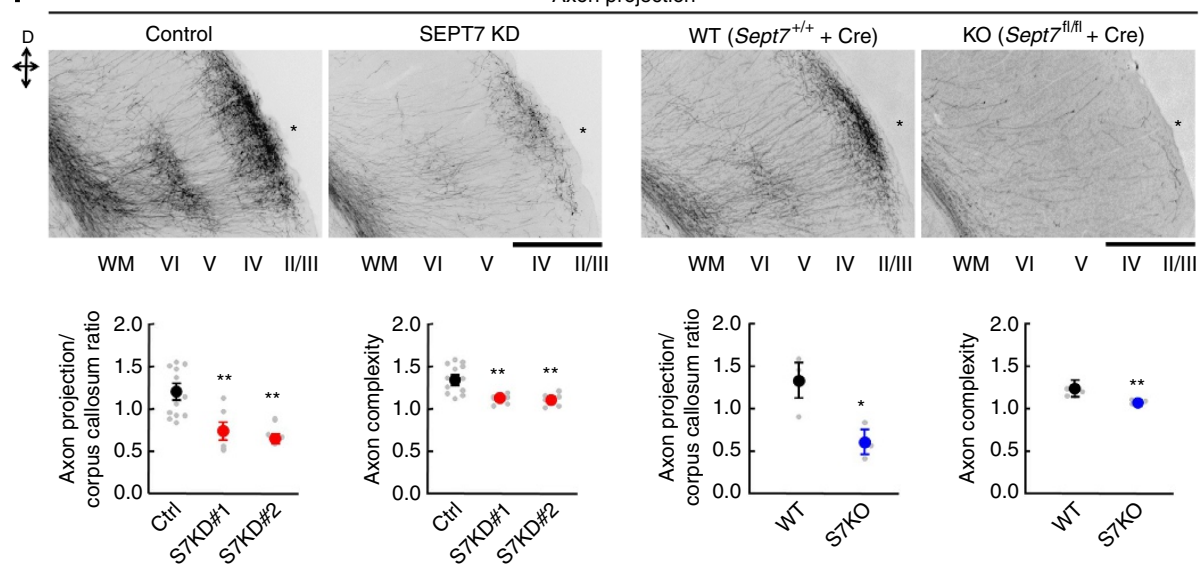

Figure 1 | The core septin subunit SEPT7 is required for the growth of dendrites and axons of cerebrocortical neurons in vivo. (a) Representative immunofluorescence images of primary cerebrocortical neurons at div2 co-expressing GFP with control (left) or shRNA\#1 against SEPT7 (right). Endogenous SEPT7 (red) is depleted in GFP-positive transfectants (green). Scale bar, $10 \mu \mathrm{m}$. Graph: SEPT7 depletion efficiency (SEPT7/GFP immunofluorescence ratio) via RNAi (S7KD\#1, \#2) or acute gene disruption (S7KO). ( $n=12 .{ }^{\star \star \star} P<0.001$ by one-way ANOVA with post hoc Tukey for KD, $t$-test for KO). Error bars denote s.e.m. (b) SEPT7 depletion efficiency (SEPT7/ $\alpha$-tubulin immunofluorescence ratio) in vivo via RNAi (S7KD\#1). FACSsorted GFP-positive neurons from P2 cerebral cortices were immunoblotted. Triplicated experiments. ( ${ }^{\star \star \star} P<0.001$ by $t$-test. See Supplementary Fig. S2). Error bars denote s.e.m. (c) P15 mouse coronal section with a layer II/III pyramidal neuron in the somatosensory cortex (S1/S2). Prospective layer II/III pyramidal neurons were subjected to KD/KO at E15 and analysed at P15 for the dendrites (d), callosal axon bundles (e) and axon terminals in the contralateral S1/S2 (f). (d) Representative projected images of GFP-positive layer II/III pyramidal neurons. Scale bars, $50 \mu \mathrm{m}$. Graph: SEPT7 depletion via $\mathrm{KD} / \mathrm{KO}$ consistently reduced the total length and tip number of dendrites. (KD; $n=18,12,12 .{ }^{\star \star \star} P<0.001$ by one-way ANOVA with post hoc Tukey. $\mathrm{KO} ; n=7,{ }^{\star \star \star} P<0.001, t$-test). Error bars denote s.e.m. (e) Representative images of GFP-positive axon bundles at the corpus callosum. Scale bar, $250 \mu \mathrm{m}$. Graph: SEPT7 depletion did not affect the axon projection up to the midline. (KD; $n=12,6,6$. NS, P>0.05 by one-way ANOVA with post hoc Tukey. KO; $n=3$. NS, $P>0.05$ by $t$-test). Error bars denote s.e.m. (f) Representative images of GFP-positive axon terminals projecting toward the contralateral S1/S2 (asterisk). The axon terminals branch and terminate at layers V and II/III. Layers (II/III to VI) and white matter (WM) are labelled at the bottom. Scale bars, $500 \mu \mathrm{m}$. Graph: GFP-fluorescence intensity in layers II/III normalized to the corpus callosum and the axon complexity at a distal segment (the ratio of the number of branches that entered layer IV over that at layer V) were significantly diminished in SEPT7-depleted neurons. Thus, the terminal extension and branching of axons were consistently impaired via RNAi and gene disruption. (KD; $n=12,6,6 .{ }^{\star \star} P<0.01$ by one-way ANOVA with post hoc Tukey. KO; $n=3 .{ }^{\star \star} P<0.01,{ }^{\star} P<0.05$ by $t$-test). Error bars denote s.e.m.

electroporation at an earlier stage (E12) into another subset of neurons to form the primary motor cortex (M1) impaired the corticospinal tract development by affecting the axon projection from the layer $\mathrm{V}$ primary motor neurons (Supplementary Fig. S5).

To corroborate the findings obtained from the RNAi experiments, we disrupted the Sept7 gene by creating mice harbouring a Cre-loxP-based conditional knockout (floxed) allele, Sept $7^{f l}$. Two days after the co-transfection of expression plasmids for Myc-Cre and GFP (as a morphological tracer) into Sept $7^{\mathrm{fl} / \mathrm{fl}}$ cerebrocortical neurons, the efficiency of SEPT7 depletion was $\sim 80 \%$ determined by the immunofluorescence of cerebrocortical neurons electroporated in vitro (Fig. 1a, right). We confirmed the comparable effects of SEPT7 depletion on dendrite growth, interhemispheric axon projection and terminal branching of layer II/III pyramidal neurons in S1/S2 (Fig. 1d, right; 1e, bottom; 1f, right).

Together, these data consistently indicate that SEPT7 is commonly required for the elongation and branching of both dendrites and axons in the developing mouse brain. This is the first evidence for the cell autonomous requirement of septins in neuritogenesis in vivo.

SEPT7 is required for dendrito- and axonogenesis in vitro. To rigorously assess the short dendrite/axon phenotype, we 
quantified the effects of SEPT7 depletion from cerebrocortical neurons via RNAi or gene disruption in vitro. Two days after transfection of the shSept7/GFP plasmid, the gross knockdown efficiency estimated by immunoblot for SEPT7 (including the GFP-negative population of 30-40\%) was $~ 30-40 \%$ (Fig. 2a and Supplementary Fig. S10), whereas the knockdown efficiency per individual GFP-positive cell estimated by immunofluorescence for SEPT7 was $\sim 80 \%$ (Fig. 1a). Each of the SEPT7-depleted pyramidal-like neurons typically bore several dendrites and an axon as did the control neurons (Fig. 2b). Morphometry that separately quantified the total lengths of dendrites and the axon of each neuron revealed that SEPT7-depleted pyramidal-like neurons bore significantly shorter dendrites (60 versus $105 \mu \mathrm{m}$ at the 50th percentile) and axons (210 versus $350 \mu \mathrm{m}$ at the 50 th percentile) with less complexity (branch tip number) than the control. Scattergram analysis indicates that depletion of SEPT7 caused proportional defects in dendrites and axons in individual neurons. The morphological phenotype was almost completely rescued by co-expressing an RNAi-resistant Sept7 mRNA with a red fluorescent protein, mCherry (Fig. 2c,d).

The findings obtained from the RNAi experiments were corroborated by Sept7 gene disruption. Two days after the coexpression of Myc-Cre and GFP into Sept $7^{\mathrm{fl} / \mathrm{fl}}$ cerebrocortical neurons, SEPT7 depletion estimated by immunoblot analysis was $\sim 50 \%$ (including a GFP-negative population of 30-40\%) (Fig. $2 \mathrm{e}$ and Supplementary Fig. S10), when Sept7-null pyramidal-like neurons bore significantly shorter dendrites (53 versus $120 \mu \mathrm{m}$ at the 50th percentile) and axons (168 versus $328 \mu \mathrm{m}$ at the 50 th percentile) with less complexity than the control (Fig. $2 \mathrm{f}-\mathrm{h}$ ). The possibility of Cre-mediated toxicity on neuritogenesis was excluded by control wild-type neurons expressing Cre.

Taken together, the consistent growth impairment of dendrites and axons after the acute partial depletion of SEPT7, either in vivo or in vitro, either via RNAi or gene disruption, indicates a

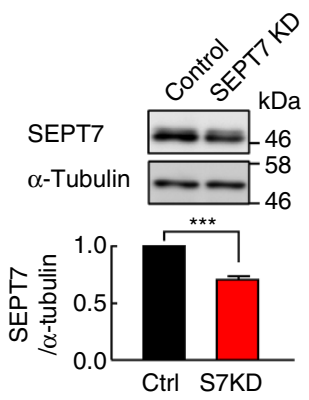

b

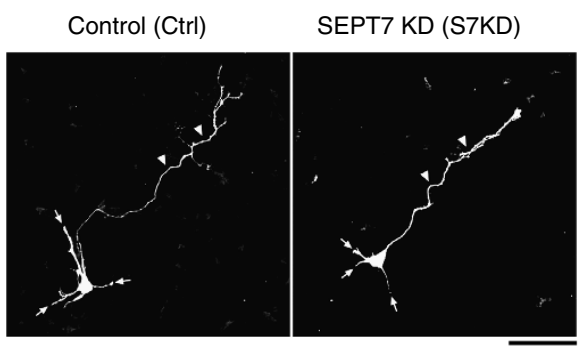

$\mathbf{f}$
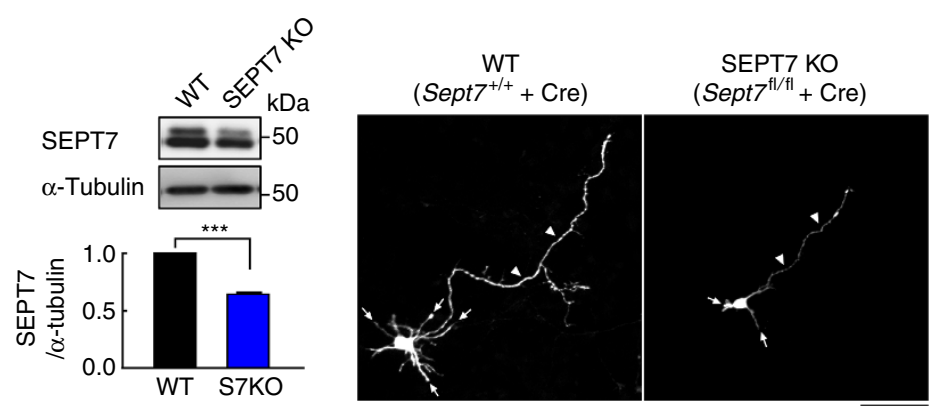

C

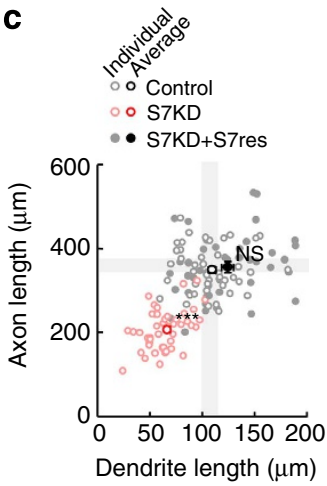

g

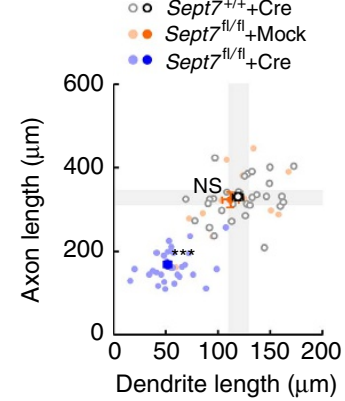

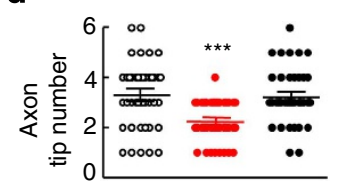

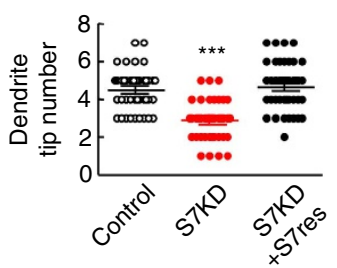

h
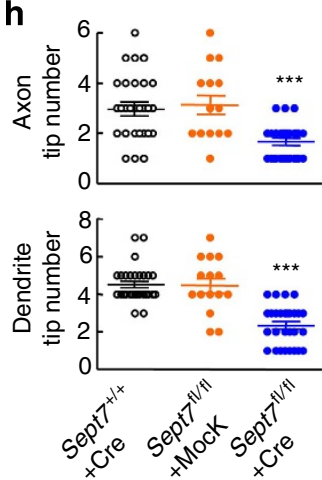

Figure 2 | In vitro rescue and morphometry of the SEPT7-depletion phenotype on dendrite/axon growth. (a) Immunoblot estimation of the gross efficiency (including GFP-negative untransfected population) of SEPT7 depletion from wild-type E17 embryo-derived neurons via S7KD\#1 RNAi (div2). Each lane contained $10 \mu \mathrm{g}$ protein. (Triplicated experiments. ${ }^{\star \star \star} P<0.001$ by $t$-test). Error bars denote s.e.m. (b) Representative images for the morphometry of GFP-positive pyramidal-like neurons with dendrites (arrows) and axons (arrowheads). Scale bar, $50 \mu \mathrm{m}$. (c) Scattergram of the total lengths of the axon and dendrites from each neuron expressing the designated plasmid(s). Dendrites and axons of SEPT7-depleted neurons (S7KD, red) were significantly and proportionally shorter than control neurons (black) whose average \pm 2 s.e.m. is indicated as grey zones. The SEPT7-depletion phenotype was rescued by the co-expression of an RNAi-resistant mRNA encoding mCherry-SEPT7 (S7KD + S7res, closed circles). $\left(n=45 \times 3 .{ }^{* \star *} P<0.001\right.$; NS, $P>0.05$ by one-way ANOVA with post hoc Tukey). Error bars denote s.e.m. (d) Significant differences in the tip numbers of axons and dendrites between the experimental groups $\left(n=45 \times 3 .{ }^{\star \star \star} P<0.001\right.$ by one-way ANOVA with post hoc Tukey), which were rescued as noted above. Error bars denote s.e.m. (e) Experiment comparable with (a), but using Sept $7^{f l / f l}$ embryo and Myc-Cre plasmid. Immunoblot estimation of the gross efficiency (including the untransfected population) of SEPT7 depletion after gene disruption (div2). See legend in (a). Error bars denote s.e.m. (f) Representative images for the morphometry of GFP/Cre-positive, Sept7 ${ }^{+/+} /$wild type (WT) and Sept $7^{\mathrm{fl} / \mathrm{fl}}$ (SEPT7KO) pyramidal-like neurons. See legend in (b). (g) Scattergram of the total lengths of the axon and dendrites from each neuron with designated genotype \pm Cre expression. Dendrites and axons of Sept $7^{\mathrm{fl} / \mathrm{fl}}+\mathrm{Cre}(\mathrm{SEPT} 7 \mathrm{KO}$, blue circles) neurons were significantly and proportionally shorter than those of control Sept $7^{\mathrm{fl} / \mathrm{fl}}-\mathrm{Cre}$ (orange circles) neurons and Sept $7^{+/+}+\mathrm{Cre}$ (open circles) neurons whose average \pm 2 s.e.m. is indicated as grey zones. Statistical analysis showed that acute Sept7 disruption significantly and proportionally shortened their dendrites and axons. $\left(n=30,15,30 .{ }^{\star \star \star} P<0.001\right.$ by one-way ANOVA with post hoc Tukey). Note that the short dendrite/ axon phenotype elicited by gene disruption was more severe than elicited by RNAi in (c). Error bars denote s.e.m. (h) The tip numbers of axons and dendrites were significantly reduced after Sept7 disruption. $\left(n=30,15,30 .{ }^{\star \star} P<0.001\right.$ by one-way ANOVA with post hoc Tukey). Error bars denote s.e.m. 
the physiological requirement of SEPT7 in a common cell autonomous mechanism that drives neurite extension.

SEPT7 limits acetylation and stabilization of microtubules. One of the common and major structural determinants of dendritogenesis and axonogenesis is the dynamic assembly of microtubules, which is regulated by a number of mechanisms including tubulin modifications ${ }^{21}$. On the basis of previous studies on aberrant microtubule modifications in septin-depleted non-neural cells (see Discussion), we examined the status of the two major microtubule modifications, acetylation and polyglutamylation, in cerebrocortical neurons in vivo and in vitro. Acetylated $\alpha$-tubulin significantly accumulated in GFP-positive/SEPT7-depleted layer II/III cerebrocortical neurons, determined by immunoblot of FACScollected neurons from E17 embryos $(\times 5$, Fig. $3 \mathrm{a}$ and
Supplementary Fig. S10), and by immunofluorescence of the somata and dendrites $(\times 9$, Fig. $3 b)$ and of axons to a lesser extent $(\times 3$, Fig. 3c). The differential acetylation levels of dendrites and axons might be correlated with the differential effects of SEPT7 depletion observed in vivo (Fig. 1d,f).

Consistently, in vitro experiments showed that the amount of acetylated $\alpha$-tubulin increased after depletion of SEPT7 either via RNAi or gene disruption. In either case, the gross increment of acetylated $\alpha$-tubulin estimated by immunoblot was $30-40 \%$ including the GFP-negative untransfected population (Fig. 3d,e; Supplementary Fig. S10), whereas the amount of polyglutamylated $\alpha$-tubulin was changed only by $-1 \%$ as compared with the control. Immunofluorescence analysis detected hyperacetylated $\alpha$-tubulin along the dendrites and axons, and in the somata of SEPT7-depleted neurons (Fig. 3f). a

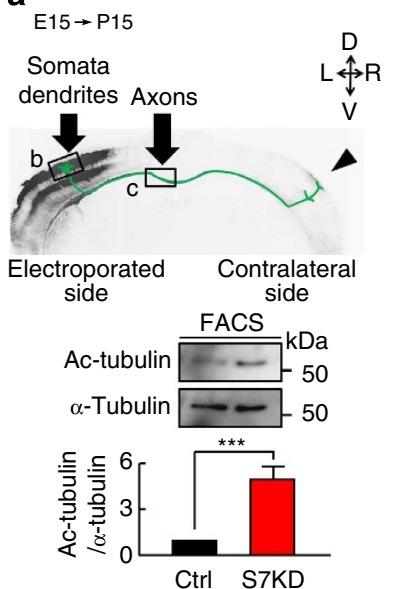

d
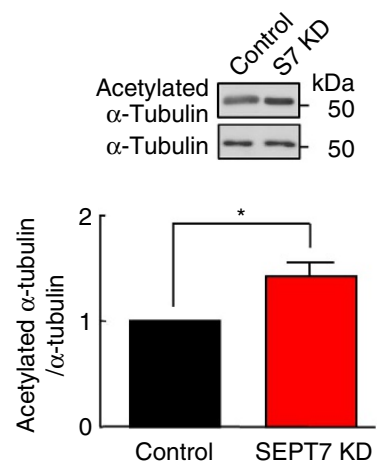

b
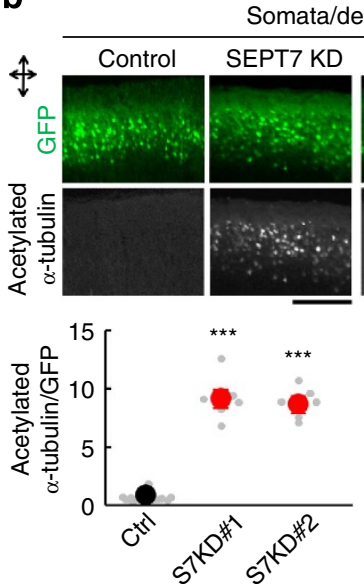

e
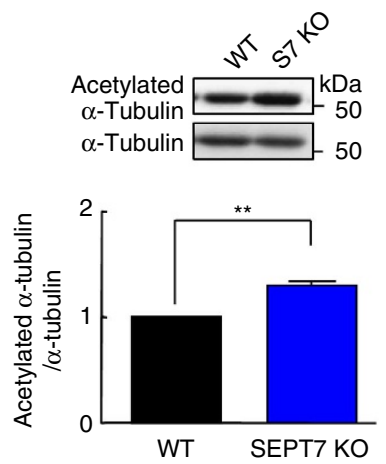
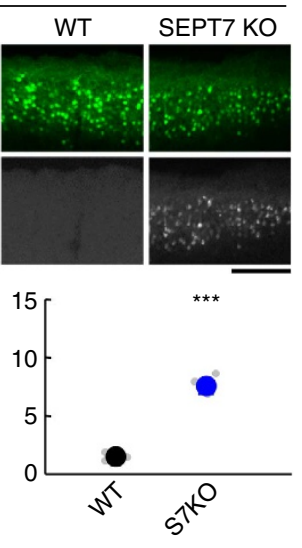

C
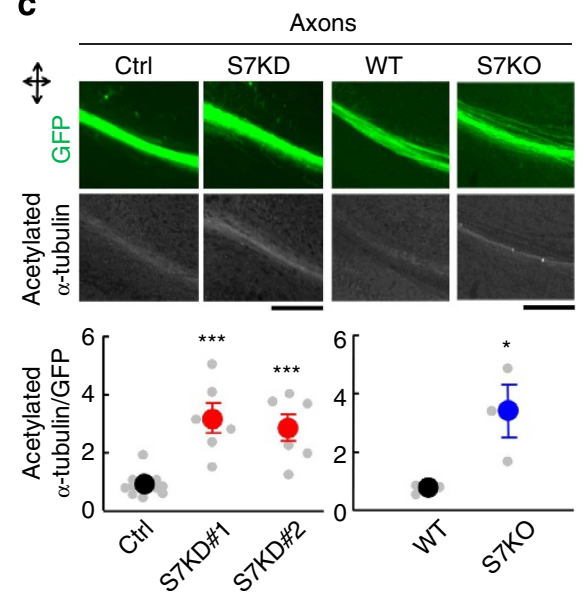

f

Figure 3 | Depletion of SEPT7 causes significant accumulation of acetylated $\alpha$-tubulin in vivo and in vitro. (a) (top) Coronal section of P15 brain depicting two subcellular segments of layer II/III pyramidal neurons in S1/S2 (modified from Fig. 1c). (bottom) FACS-sorted GFP-positive cerebrocortical neurons electroporated in utero at E15 and collected at P2 (Supplementary Fig. S2) were immunoblotted for total and acetylated $\alpha$-tubulin. Depletion of SEPT7 by RNAi caused significant acetylation of $\alpha$-tubulin. (Triplicated experiments. ${ }^{\star \star \star} P<0.001$ by $t$-test). Error bars denote s.e.m. (b,c) Image-based quantification of acetylated $\alpha$-tubulin in the somata and dendrites and the axon bundle proximal to the corpus callosum. (top) Representative immunofluorescence images of acetylated $\alpha$-tubulin in the somata/dendrites (b) and in the axon bundle (c) of GFP-positive S1/S2 pyramidal neurons with or without SEPT7 depletion via RNAi (\#1) or gene disruption. In SEPT7-depleted neurons, microtubules were significantly acetylated in the somata/dendrites and in the axons to a lesser extent. Scale bars, $500 \mu \mathrm{m}$ (b), $200 \mu \mathrm{m}$ (c). Graphs: Acetylated $\alpha$-tubulin/GFP immunofluorescence ratio in vivo via RNAi (S7KD\#1 and \#2) or gene disruption (S7KO). All three modes of SEPT7 depletion consistently caused significant acetylation of $\alpha$-tubulin in the somata/dendrites and in the axons to a lesser extent. (KD; $n=12,6,6 .{ }^{* \star \star} P<0.001$ by one-way ANOVA with post hoc Tukey. KO; $n=3,{ }^{\star \star \star} P<0.001,{ }^{\star} P<0.05$ by $t$-test). Error bars denote s.e.m. (d,e) Immunoblot estimation of the gross acetylated/total $\alpha$-tubulin ratio (including GFP-negative untransfected population) in SEPT7-depleted cerebrocortical neurons via RNAi (\#1, KD) or gene disruption (KO) at div2. Note that $\alpha$-tubulin was hyperacetylated in SEPT7-depleted neurons. (Triplicated experiments. ${ }^{\star} P<0.05,{ }^{\star \star} P<0.001$ by $t$-test). Error bars denote s.e.m. (f) (top) Representative immunofluorescence images of acetylated $\alpha$-tubulin in pyramidal-like cerebrocortical neurons in culture (div2). The insets show GFP co-expressed with shSept7(\#1) or the empty vector. The signals for acetylated $\alpha$-tubulin were elevated in the soma, dendrites and axons of SEPT7-depleted neurons (SEPT7 KD). Scale bars, $50 \mu \mathrm{m}$. (bottom) Quantification of acetylated $\alpha$-tubulin immunofluorescence measured separately in dendrites and axons, showing that SEPT7 depletion caused hyperacetylation of microtubules in axons and dendrites to a comparable degree. $\left(n=12,{ }^{\star \star \star} P<0.001,{ }^{\star \star} P<0.01\right.$ by $t$-test). Error bars denote s.e.m. 
To assess the growth rate of axonal microtubules following SEPT7 depletion, we conducted live imaging of the plus ends of microtubules with GFP-EB1 in div3 (1 day after plating) cerebrocortical neurons from E17 mice. The average anterograde velocity of GFP-EB1 puncta was significantly reduced in the longest neurites (presumed axons) of neurons that co-express $\operatorname{shSept7(\# 1)/mCherry~compared~with~those~that~express~mCherry~}$ alone (Fig. 4a-d; Supplementary Movies 1 and 2). The range of GFP-EB1 velocity in the control group was comparable with the previous results on EB3-GFP in axons ${ }^{22}$, which guarantees the authenticity of our experimental conditions and neuronal viability. These data indicate that the retardation of microtubule growth underlies the stagnant neurite growth after depletion of SEPT7.

The consistent in vivo and in vitro findings indicate that depletion of SEPT7 from cerebrocortical neurons causes hyperacetylation of microtubules via functional insufficiency of HDAC6. The resulting growth retardation and hyperstabilization (Supplementary Fig. S6) of microtubules ${ }^{23}$ are likely to account, at least in part, for the reduced neuritogenesis elicited by the loss of SEPT7.

Septins and HDAC6 are associated in vivo and in vitro. We reasoned that SEPT7 may promote the deacetylation or suppress the acetylation of $\alpha$-tubulin via an unknown mechanism. On the basis of previous studies, we tested the possible involvement of

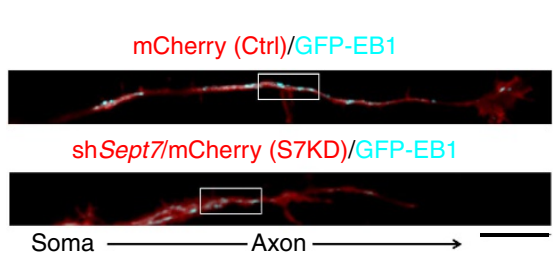

b

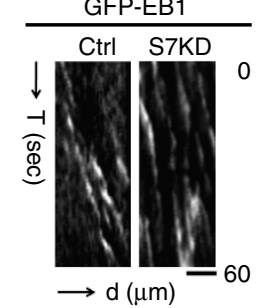

C
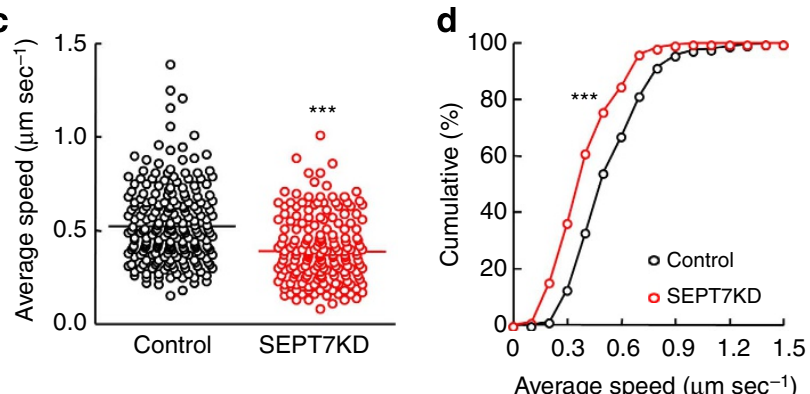

Figure 4 | Stagnant microtubule growth in SEPT7-depleted cerebrocortical neurons. (a) Representative frames from time-lapse images of GFP-EB1 (cyan), a microtubule plus end tracker, expressed in cerebrocoritical pyramidal-like neurons at div3 (1 day after plating) with or without SEPT7 depletion via RNAi (\#1). Each image shows a part of a transfected neuron expressing mCherry whose longest neurite (presumed axon) extends rightward from the soma. Scale bar, $10 \mu \mathrm{m}$. (b) Kymographic representation of the anterograde motility of GFP-EB1 puncta. Time-lapse serial images of GFP-EB1 in an axon segment (boxed in a) are stacked. The slope of the trajectory represents the velocity of each GFP-EB1 punctum. Scale bar, $5 \mu \mathrm{m}$. (c,d) Anterograde velocity of GFP-EB1 puncta measured in the longest neurites, with or without SEPT7 depletion, and shown as a scattergram (c) and as cumulative curves (d). SEPT7 depletion (S7KD, red) reduced the fast-moving population and shifted the curve to the left. The velocity was measured for 217 (Ctrl) and 188 (S7KD) GFP-EB1 puncta that were tracked for more than $10 \mathrm{~s}$, respectively in 18 and 16 mCherryexpressing neurons that were morphologically comparable. ${ }^{\star \star \star} P<0.001$ by $t$-test in (c) and by the Kolmogorov-Smirnov test in (d)). the major $\alpha$-tubulin deacetylase in neurons, HDAC6 (refs 24-26). After confirming the expression of HDAC6 in the newborn (P0) cerebral cortices and cerebrocortical neurons at div2-3 (Fig. 5a,b; Supplementary Fig. S10), we tested whether HDAC6 and septins are associated in extracts of those cells using a co-immunoprecipitation/immunoblot assay. The reciprocal co-immunoprecipitation between subsets of endogenous SEPT7 (in complex with other subunits) and HDAC6 with specific antibodies suggests their physiological association during neuritogenesis (Fig. $5 \mathrm{c}$ and Supplementary Fig. S10). To test the association between exogenous SEPT7 and HDAC6, we co-expressed epitope-tagged polypeptides in heterologous cells, and showed that GFP-tagged SEPT7 is captured on anti-Flag antibody-coated beads via Flagtagged HDAC6 (Fig. 5d and Supplementary Fig. S10). We further tested the direct interaction between the SEPT7-containing septin complex and HDAC6 in vitro. $\mathrm{His}_{6}$-tagged SEPT7/6/2 (a canonical septin hetero-oligomer used due to the higher solubility than SEPT7 alone ${ }^{27}$ ) that had been expressed in insect cells, then purified and immobilized on beads, captured bacterially expressed recombinant GST-tagged HDAC6 but not GST-tag alone (Fig. 5e and Supplementary Fig. S10). These data collectively support the direct interaction between the SEPT7-containing septin complex and HDAC6. In contrast, the association between SEPT7 and another $\alpha$-tubulin deacetylase SIRT2 (refs 25,26), or two major $\alpha$-tubulin acetyl transferases MEC-17 (ref. 28) and ELP3 (ref. 29), was not detectable in cerebrocortical neurons (Supplementary Fig. S7).

To visualize the subcellular location where SEPT7 and HDAC6 associate, we used the in situ proximity ligation assay (PLA) ${ }^{30}$ for sprouting cerebrocortical neurons in culture. In theory, a pair of antibodies that recognize endogenous SEPT7 and HDAC6 in close proximity $(<40 \mathrm{~nm})$ mediates a local enzymatic reaction that generates a submicron fluorescent deposit. The fluorescent puncta, which represent antibody-crosslinked SEPT7 and HDAC6, were distributed both in the soma and neurite (Fig. 5f). Taken together, the physical interaction between the SEPT7-containing septin complex and HDAC6 was consistently detected in biochemical assays and was corroborated by their proximity in situ.

SEPT7 facilitates HDAC6/acetylated $\alpha$-tubulin interaction. Given the direct molecular interaction between septins and HDAC6, and between HDAC6 and acetylated $\alpha$-tubulin as a substrate, a simple mechanism connecting the depletion of SEPT7 to microtubule hyperacetylation could involve the collateral depletion and/or inhibition of HDAC6. However, this scheme seems unlikely, as depletion of SEPT7 did not significantly alter the amount of HDAC6 or its enzymatic activity on a fluorogenic substrate (Fig. 5g,h; Supplementary Figs S8 and S10). Intriguingly, despite the accumulation of acetylated $\alpha$-tubulin in SEPT7-depleted cells (Fig. 3d,e), the HDAC6-bound fraction of acetylated $\alpha$-tubulin was paradoxically reduced (Fig. $5 \mathrm{i}$ and Supplementary Fig. S10). This discordance indicates a reduced accessibility of HDAC6 to acetylated $\alpha$-tubulin in the absence of SEPT7, and favours another scheme: Interaction with the septin complex may enhance the accessibility of HDAC6 to acetylated $\alpha$-tubulin, possibly by hindering the access of competitive binding partners that include substrates of HDAC6.

Inhibition of HDAC6 gives a phenocopy of SEPT7 depletion. To probe the functional interaction between SEPT7 and HDAC6, we tested whether inhibiting microtubule deacetylation by HDAC6 could mimic and/or synergize the effect of SEPT7 depletion on neuritogenesis of cerebrocortical neurons in vitro. The deacetylation activity of HDAC6 was directly inhibited by a 

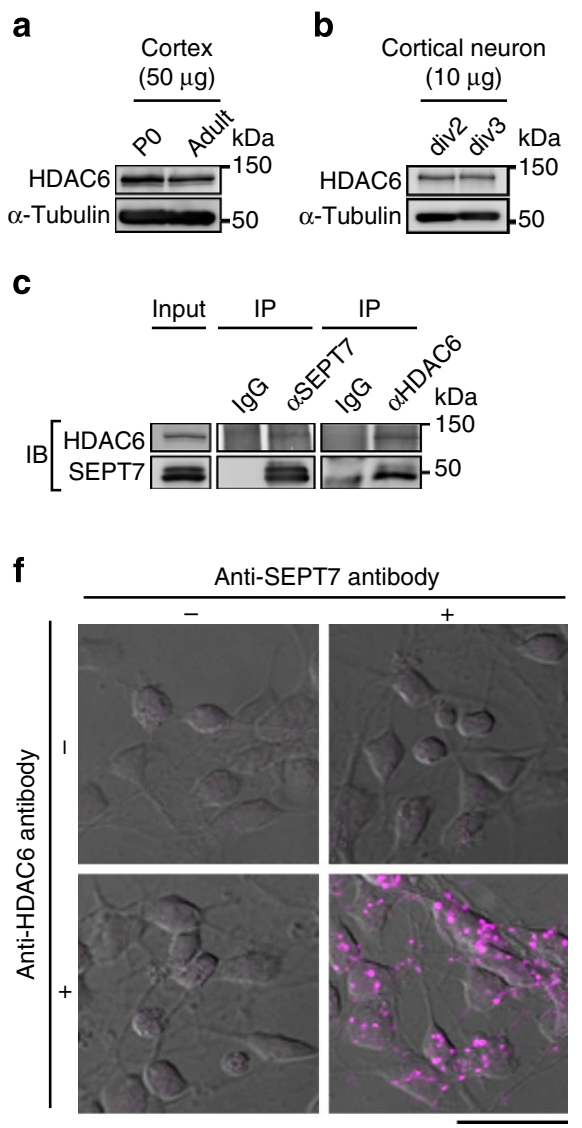

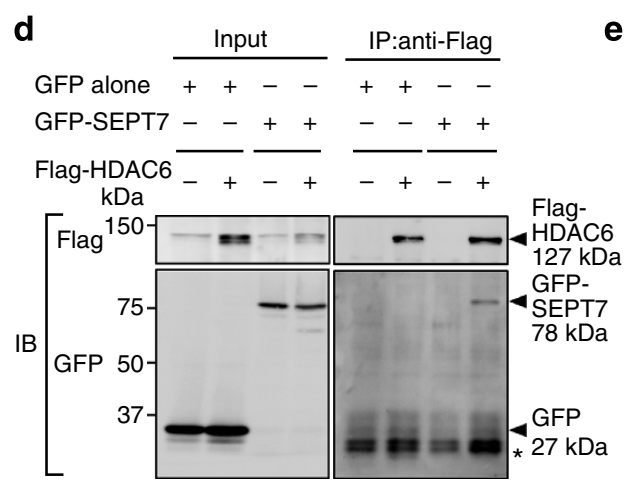

g
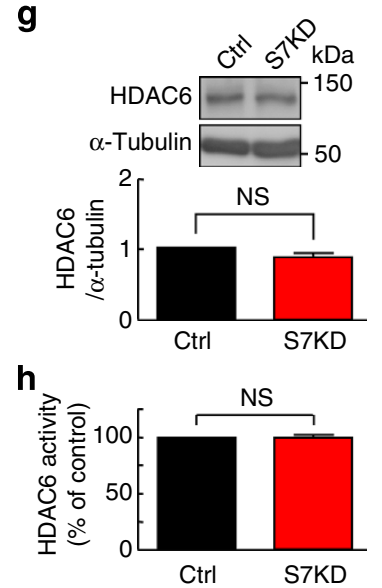

i
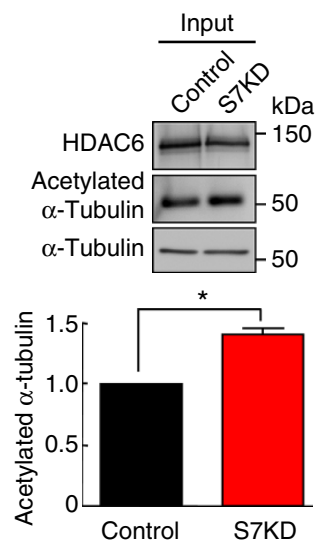

IP:

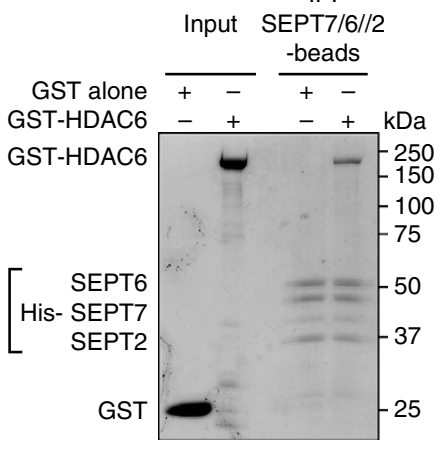

IP: Anti-HDAC6
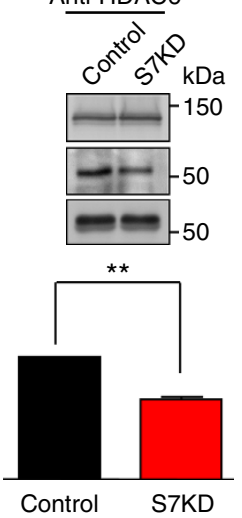

Figure 5 | Physiological and direct interaction between septins and the major tubulin deacetylase HDAC6. (a,b) Immunoblot for endogenous HDAC6 (a) in newborn (PO) and adult cerebral cortex and (b) in primary cerebrocortical neurons at div2 and 3 . Each lane respectively contained $50 / 10 \mu g$ total protein. (c) Mutual co-immunoprecipitation of endogenous SEPT7 and HDAC6. Lysates from div2 cerebrocortical neurons were incubated with protein A beads coated with nonimmune IgG, anti-SEPT7 antibody or anti-HDAC6 antibody, and these proteins were detected by immunoblot in a reciprocal manner. (d) Co-expression and mutual co-immunoprecipitation of GFP-SEPT7 and Flag-HDAC6 in heterologous cells. (Left) Anti-Flag antibody detected FlagHDAC6 (and gave nonspecific faint bands in the 1st and 3rd lanes) and anti-GFP antibody detected GFP and GFP-SEPT7. (Right) When Flag- HDAC6 was captured on anti-Flag beads, GFP-SEPT7 was co-immunoprecipitated, but GFP was not (compare the 2nd and 4th lanes. *, IgG light chain). (e) Direct binding between the purified, recombinant septin complex and HDAC6 in vitro. Immobilized His-tagged SEPT7/6/2 captured GST-HDAC6 but not GST alone. (Coomassie blue staining). (f) Representative results of in situ proximity ligation assay for endogenous SEPT7 and HDAC6 in div2 cerebrocortical

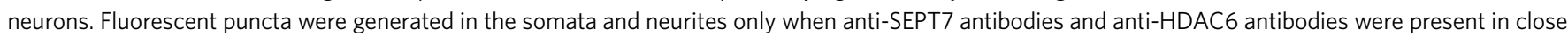
proximity. Scale bars, $25 \mu \mathrm{m}$. (g) Immunoblot showing that SEPT7 depletion did not affect the amount of HDAC6 in div2 cerebrocortical neurons. (Triplicated experiment. NS, P>0.05 by t-test). Error bars denote s.e.m. (h) SEPT7 depletion via RNAi (\#1, S7KD) did not alter the deacetylating activity of

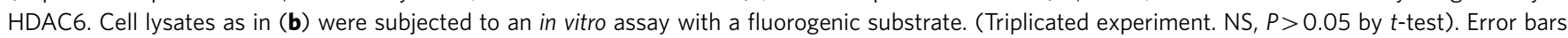
denote s.e.m. (i) SEPT7 depletion via RNAi (\#1, S7KD) caused dissociation of HDAC6 and acetylated $\alpha$-tubulin. (Left) Cell lysates as in (b,h) were immunoblotted for endogenous HDAC6, acetylated $\alpha$-tubulin and total $\alpha$-tubulin. Hyperacetylation of $\alpha$-tubulin in SEPT7-depleted neurons was

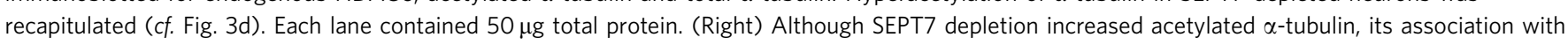
HDAC6 was paradoxically reduced. (Triplicated experiments. ${ }^{\star} P<0.05,{ }^{\star \star} P<0.01$ by $t$-test). Error bars denote s.e.m.

selective, cell-permeable HDAC6 inhibitor, tubacin (tubulin acetylation inducer $)^{31}$. Treatment of cerebrocortical neurons with $\overline{10} \mu \mathrm{M}$ tubacin (from $6 \mathrm{~h}$ after plasmid transfection through to div2) significantly increased the level of acetylated $\alpha$-tubulin without altering the level of total $\alpha$-tubulin, raising the acetylated/ total $\alpha$-tubulin ratio by $34 \%$. Morphometry, scattergram and statistical analyses revealed that tubacin suppressed the growth of both dendrites and axons more potently than did the depletion of SEPT7 via RNAi (\#1), and the combination of those two genetic/ chemical techniques did not give additive effects (Fig. 6a-e). The potent inhibition of neuritogenesis by tubacin corroborates that HDAC6 is the critical $\alpha$-tubulin-deacetylating enzyme in sprouting cerebrocortical neurons.

Taken together, we conclude that the functional insufficiency, without inhibiting the enzymatic activity, of HDAC6 underlies the short neurite phenotype found in SEPT7-depleted neurons, and that HDAC6-mediated modification of microtubules is a major biochemical process that connects the gap between the loss of SEPT7 and neuritogenesis defects in vivo and in vitro.

\section{Discussion}

In this study, we provide the most compelling evidence to date demonstrating the cell autonomous requirement of the septin subunit SEPT7 for dendrite growth and terminal extension/ branching of axons of cerebrocortical neurons in pre- and postnatal mouse brain development. The results were consistent regardless of the means used to deplete SEPT7 (RNAi and conditional Sept7 gene disruption) and of the system (in vivo and in vitro). Biochemical, pharmacological and imaging analyses 
a

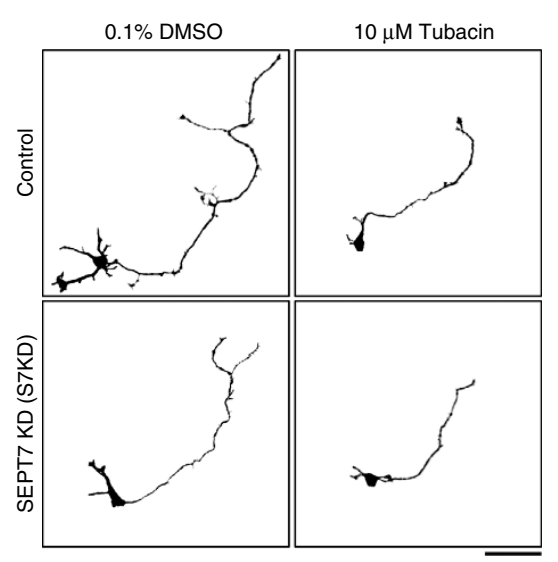

b
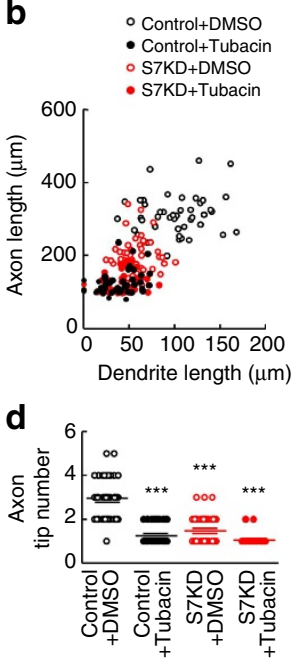

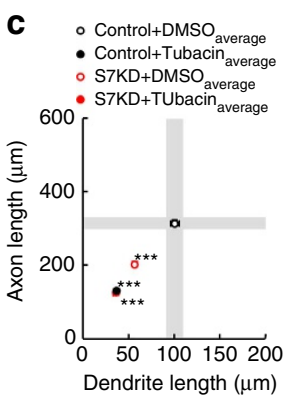

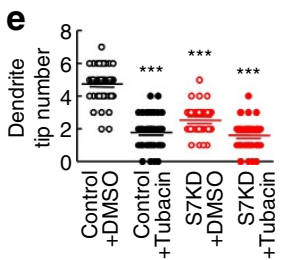

Figure 6 | Direct inhibition of HDAC6 by tubacin gives a phenocopy of SEPT7 depletion. (a) (Top panels) Representative images of GFP-expressing cerebrocortical neurons at div2 treated with vehicle alone (0.1\% DMSO) or with $10 \mu \mathrm{M}$ tubacin in vehicle. HDAC6 inhibition by tubacin inhibited the growth of both dendrites and axons. (Bottom panels) SEPT7 depletion via RNAi (\#1) combined with tubacin treatment did not exhibit an obvious additive effect on neurite morphology. Scale bar, $50 \mu \mathrm{m}$. (b) Scattergram of total dendrite length and total axon length of pyramidal-like cerebrocortical neurons treated with tubacin and SEPT7-depletion in the four combinations shown in (a). The growth of both dendrites and axons was markedly inhibited by tubacin treatment (black closed circles) in comparison with the control (black open circles), which was not enhanced by additional depletion of SEPT7 (red closed circles). (c) Statistical analysis of the results shown in (b) with plots of the mean values, showing that tubacin treatment significantly and proportionally shortened dendrites and axons. The neurite-shortening effect of tubacin was more potent than, and not enhanced by, SEPT7 depletion. The grey bars represent average \pm 2 s.e.m. of the control samples. $\left(n=45 \times 4\right.$. ${ }^{\star \star \star} P<0.001$ by one-way ANOVA with post hoc Tukey). Error bars denote s.e.m. (d and e) Tip numbers of axons and dendrites in the above samples were significantly reduced by tubacin alone, by SEPT7 depletion alone, or by the combination of the two. $\left(n=45 \times 4 .{ }^{\star \star \star} P<0.001\right.$ by one-way ANOVA with post hoc Tukey). Error bars denote s.e.m.

uncovered that septins/HDAC6-mediated deacetylation of microtubules is the common mechanism that couples septins and the dynamic growth of microtubules, shared by the dendrites and axons of sprouting neurons (Fig. 7).

Previously, the functional role(s) of septins in neurons had remained controversial: RNAi in cultured neurons gave morphological phenotypes such as dendrite malformation of rat hippocampal neurons ${ }^{14,15}$ or aberrant axon branching of chick dorsal root ganglia neurons ${ }^{16}$. In contrast, no gross neuroanatomical deficit was reported in conventional knockout mice that lack SEPT3, $4,5,6,3+5$ or $4+6$ (refs $7-11$ ). This led to the notion that perhaps septins might be dispensable as cytoskeletal or structural components in neurons, but that septins might rather compartmentalize and/or coordinate proper targeting/ complexing of signalling molecules and transporters ${ }^{10,32-34}$. Our observations indicating that SEPT7 facilitates the access of HDAC6 to acetylated $\alpha$-tubulin reconcile the seemingly contradictory observations and underscore the critical role of septins as a scaffold for microtubule regulation.

A previous study with the dog kidney-derived MDCK cell line showed that the non-neuronal septin subunit SEPT2 is associated with polyglutamylated microtubules ${ }^{35}$, a subset of which is required to guide microtubule growth ${ }^{36}$. However, in cerebrocortical neurons, the amount of polyglutamylated $\alpha$-tubulin changed only by $-1 \%$ after the depletion of SEPT7. In HeLa cells, depletion of SEPT7 or SEPT2 releases a septin binding partner, MAP4, and the resulting relocation of MAP4 causes hyperstabilization of microtubules ${ }^{37}$. In neurons, however, MAP4 is much lower in abundance than the major microtubuleassociated proteins, MAP2 and tau. Thus, the previously reported molecular mechanisms that mediate septins and microtubules in heterologous cells are unlikely to have major roles in neuritogenesis.
The physical interaction between septins and HDAC6 is consistent in three distinct biochemical assays; first, co-immunoprecipitation of native SEPT7 and HDAC6 from extracts of cerebrocortical neurons; second, co-immunoprecipitation of SEPT7 and HDAC6 co-expressed in nonneural cells; and finally, beads-capture assay with a pure recombinant SEPT7/6/2 complex and HDAC6. SEPT7-HDAC6 interaction was less obvious in the first than in second or third assay, implying that the interaction is subject to their biochemical states (for example, post-translational modifications of HDAC6 and the composition of septin heterooligomers) and/or the environment (for example, competition with other interaction partners expressed in neurons).

HDAC6, which belongs to a class II histone deacetylase family, is a multidomain deacetylase/adaptor protein with tandem catalytic domains and a ubiquitin-binding zinc finger. HDAC6 interacts with and exerts pleiotropic effects on or with diverse proteins, which are categorized into at least two classes; (1) deacetylation substrates (for example, $\alpha$-tubulin, HSP90, cortactin), and (2) adapters (for example, $\beta$-tubulin, p150 glued/dynactin, Cdc20, polyubiquitin) ${ }^{38}$. Given the multiple binding partners of HDAC6 and the fact that depletion of SEPT7 reduces HDAC6bound acetylated $\alpha$-tubulin without affecting HDAC6 activity in neurons, a simple hypothesis would be that septins facilitate the interaction between acetylated $\alpha$-tubulin and HDAC6 by hindering the access of competing binding partners or substrates to HDAC6. This model may account for the requirement of septins to maintain microtubule dynamics for neurite growth through HDAC6-mediated deacetylation. That model is supported by the facts that another $\alpha$-tubulin deacetylase SIRT2 (refs 25,26 ), acetyltransferase elongator complex components MEC-17 (ref. 28) and ELP-3 (ref. 29) do not interact with SEPT7, and that their amounts do not change by depletion of SEPT7 (Supplementary Fig. S7), although other possibilities are not excluded. 


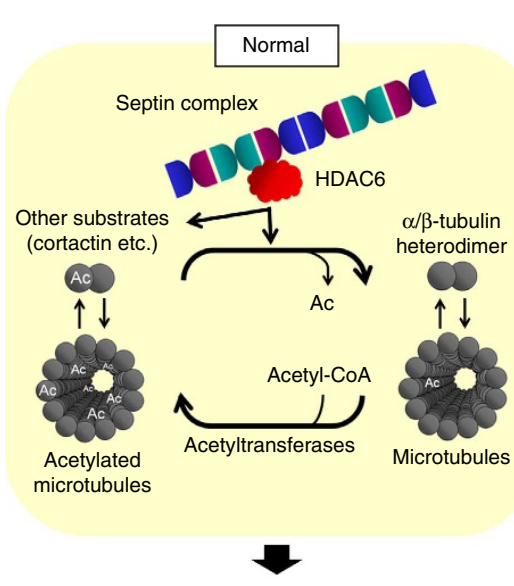

Dynamic microtubules

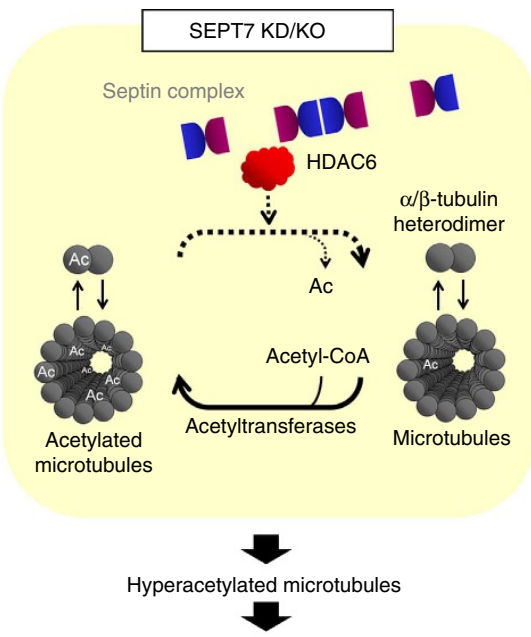

Suppression of microtubule dynamics

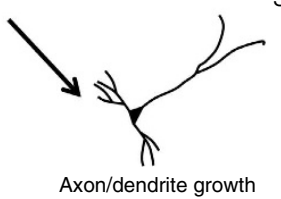

Figure 7 | Schematic diagram illustrating the involvement of the HDAC6-septin association in microtubule deacetylation and axon/dendrite growth. HDAC6 is the major microtubule deacetylase, which promotes microtubule remodelling by counteracting acetyl transferases that stabilize microtubules ${ }^{55}$. This study demonstrated that the direct interaction with the septin complex facilitates the access of HDAC 6 to acetylated $\alpha$-tubulin and/or stabilizes the enzyme-substrate interaction without altering the deacetylation activity of HDAC6. An open question is whether the putative tripartite interaction of the septin complex/HDAC6/acetylated $\alpha$-tubulin (a subset of the signals in the PLA assay shown in Fig. 5 f should represent this) occurs on $\alpha / \beta$-tubulin heterodimers, protofilaments and/or microtubules. It is also worth testing whether an actomyosin-dependent mechanism, including another HDAC6 substrate cortactin ${ }^{16}$, could contribute to the stagnant neurite growth after septin depletion. Independent of the specific underlying mechanism, the novel molecular network identified in this study has shed new light on the common machinery for the growth of axons and dendrites in vivo and in vitro.

This study has disclosed a novel molecular link integrating septins, microtubules and HDAC6, a critical protein-modifying enzyme that couples the two cytoskeletal systems for the coordination of neurite growth during brain development. As several septin subunits are significantly accumulated in postmortem human brains with psychiatric disorder ${ }^{39}$ and Hdac6null mice exhibit hyperactivity and resistance against anxiety/ depression $^{40-42}$, post-developmental function and neuropathological involvement of the septin-HDAC6-microtubule axis await further examination.

Previous studies have demonstrated that Hdac6-null mice do not develop an obvious brain anomaly ${ }^{40}$, and that genetic or chemical inhibition of HDAC6 delays the progression of mouse models of adult-onset neurodegeneration, for example, CharcotMarie-Tooth disease (HSPB1-1- $)^{43}$ and amyotrophic lateral sclerosis $\left(S O D 1^{\mathrm{G} 93 \mathrm{~A}}\right)^{44}$. However, it remains unclear whether an HDAC6-targeting therapeutic regimen would be beneficial in the context of the developing brain and in adult neurogenesis, because acute depletion of HDAC6 via RNAi causes severe defects in dendritogenesis and axonogenesis in vitro and in vivo ${ }^{45,46}$. Our present study, which aimed at understanding the role of SEPT7, has provided further evidence for the developmental stage-specific requirement of HDAC6 in early neuritogenesis, and uncovered the SEPT7-HDAC6-microtubule axis as a novel molecular mechanism underlying neurite development. As the neuritogenesis phenotype was more pronounced through acute HDAC6 inhibition than through acute SEPT7 depletion (Fig. 6), future studies must address whether some forms of initial neuritogenesis defects may have been obscured by compensation in the Hdac6-null mice.

Considering the physical and functional interaction between septins and HDAC6 in mammalian neurons, pleiotropic phenotypes caused by the loss or excess of septins could be attributed partly to HDAC6 insufficiency or dysregulation, and vice versa. For instance, the loss of SEPT4 in mice diminishes resistance against $\alpha$-synuclein toxicity and dopaminergic function ${ }^{10}$, whereas HDAC6 protects Drosophila dopamine neurons from $\alpha$-synuclein toxicity ${ }^{47}$. Future studies along these lines should help elucidate the composite dysregulation of septins, HDAC6 and microtubules that may underlie the pathophysiology of developmental, psychiatric and neurodegenerative disorders in humans.

\section{Methods}

Plasmid constructions. One of the RNAi vectors used, pmU6pro-SEPT7, had been designed for the targeting of nucleotides 1005-1024 (\#2) of mouse Sept7

(ref. 27). The other RNAi vectors targeting nucleotides 1175-1196 (\#1) were constructed in UI4_GFP_SIBR or UI4_mCherry_SIBR ${ }^{20}$, each of which co-expresses shRNA and GFP or mCherry. The RNAi-resistant SEPT7 mutant (SEPT7res) was generated by introducing silent mutations into the sequence \#1 in pEGFP-SEPT7 (ref. 15), which was transferred into the pmCherry vector (a gift from Dr R.Y. Tsien at $\mathrm{UCSD} / \mathrm{HHMI}^{48}$ ) to generate pmCherry-SEPT7res. Plasmids for a Flag-tagged HDAC6 (\#13823) and a Myc-tagged Cre (\#13775) were obtained from Addgene.

Animal experiments and establishment of Sept $\mathbf{7}^{\mathrm{fl} / \mathrm{fl}}$ mice. Animal experiments were approved by the Institutional Review Committee and conducted in accordance with the regulations for the care and use of experimental animals at Nagoya University. Conditional targeting of Sept7 (exon 3) was conducted by homologous recombination with $\mathrm{C} 57 \mathrm{BL} / 6$-derived Bruce 4 embryonic stem cells, verified by Southern blot and PCR of genomic DNA. Myc-tagged Cre was introduced into $\operatorname{Sep} t 7^{\mathrm{fl} / \mathrm{fl}}$ neurons via electroporation. The recombination and SEPT7 protein decay were verified by PCR and immunoblot assays, respectively. This mouse line has been deposited to RIKEN Bioresource Center (RBRC05390).

In vivo assays. In utero electroporation was conducted by injecting a mixture of UI4_GFP_SIBR- or pmU6pro-derived constructs (for Sept $7^{+1+}$ embryos) or Myc-Cre (for $S e p t 7^{\mathrm{fl} / \mathrm{fl}}$ embryos), plus pCAG-EGFP and $0.05 \%$ Fast Green (tracer) into the lateral ventricles of embryos at E12/15. Pregnant mice were anaesthetized 
with intraperitoneal injection of sodium pentobarbital $\left(50 \mu \mathrm{gg}^{-1}\right.$, Nembutal, Abbott Laboratories) in saline. Embryos were approached through the uterus pulled out from midline laparotomy. Glass capillary tubes (GC150TF-10; Harvard Apparatus) were tapered using a micropipette puller (Narishige). One microliter of plasmid solution $\left(0.5-1.0 \mathrm{gl}^{-1}\right)$ was injected into the right lateral ventricle of each embryo, and 5 square electric pulses $(50 \mathrm{mV}, 50 \mathrm{msec})$ were given in $5 \mathrm{sec}$ with an electroporator (CUY21EDIT; NepaGene) ${ }^{19}$. Serial sagittal sections of the upper spinal cord $(30 \mu \mathrm{m}$-thick) and/or serial coronal brain sections (50 $\mu \mathrm{m}$-thick) were prepared at $\mathrm{P} 1 / 14 / 15 / 30$.

Quantitative image analyses, including the morphology-based identification of axons/dendrites (Supplementary Fig. S9), were conducted by using software MetaMorph (6.3r7, Molecular Devices). For the measurement of dendrites in the brain, GFP-positive dendrites from pyramidal neurons in the layer II/III of S1/S2 were traced in $1 \mu \mathrm{m}$-thick serial confocal sections. After assigning all dendrites to each soma, their total lengths projected onto a two-dimensional plane were measured.

FACS analysis. Six days after in utero electroporation of plasmids for RNAi, cerebral cortical tissues of P2 pups (mixed sex) were dissected and dispersed with an enzyme-free cell dissociation buffer (GIBCO). A flow cytometer (FACS Aria2, $\mathrm{BD})$ was used to collect well-separated, propidium iodide-negative (viable), and GFP-positive cells.

Cell culture. Cerebrocortical neurons from embryos were prepared and cultured using an established protocol ${ }^{19,49}$. Briefly, dissected cerebral cortices were incubated for $10 \mathrm{~min}$ with $10 \mathrm{gl}^{-1}$ trypsin type XI (Sigma) plus $0.5 \mathrm{gl}^{-1}$ DNase I type IV (Sigma) at room temperature and mechanically dissociated in Hanks solution (pH 7.4) (Sigma) with $0.5 \mathrm{gl}^{-1}$ DNase I type IV and $12 \mathrm{mM} \mathrm{MgSO}_{4}$. Dissociated cells were transfected immediately by electroporation using Nucleofector (Amaxa Biosystems) and plated onto $12 \mathrm{~mm}$ poly-L-lysine-coated coverslips at the density of $7.5 \times 10^{5}$ cells per coverslip in 24-well plates (Greiner) containing minimum essential medium (Invitrogen) with $5 \mathrm{gl}^{-1}$ glucose, $0.2 \mathrm{gl}^{-}$ $\mathrm{NaHCO}_{3}, 0.1 \mathrm{gl}^{-1}$ transferrin (Calbiochem), $2 \mathrm{mM}$ GlutaMAX-I (Invitrogen), $25 \mathrm{mgl}^{-1}$ insulin (Sigma), $1 \times$ B-27 supplement (Invitrogen), and 10\% fetal bovine serum. Tubacin ${ }^{31}$ or nocodazole (Sigma), $10 \mu \mathrm{M}$ each, was added to the medium $6 \mathrm{~h}$ after plating. HEK293T and N-18 cells ${ }^{50}$ were grown in Dulbecco's modified Eagle's medium supplemented with $10 \%$ fetal bovine serum. Cultures were maintained in $5 \% \mathrm{CO}_{2}$ at $37^{\circ} \mathrm{C}$.

Immunocytochemisty and immunohistochemistry. Cultured neurons and brain tissues were immunostained after with $4 \%$ paraformaldehyde/PBS, permeabilization with $0.1 \%$ Triton X-100/PBS, and blocking with $2 \%$ BSA/PBS $^{19,49}$. We used commercial antibodies as follows: rat or rabbit anti-GFP (1:1,000, Nacalai or Molecular Probes), rabbit anti-DsRed (1:1,000, Clontech); mouse anti-tau-1 (1:1,000, Millipore), anti-neurofilament smi312 (1:2,000, Covance), anti-MAP2 $(1: 1,000$, Chemicon), anti-Myc $(1: 1,000)$, anti-acetylated $\alpha$-tubulin $(1: 1,000)$, and anti- $\alpha$-tubulin (1:4000) (Sigma). We used the original affinity-purified rabbit polyclonal antibodies, anti-SEPT7 $(1: 4,000)^{10}$ and anti-HDAC6 (epitope: mouse HDAC6 $\left.^{1137-1149}\right)\left(1 \mathrm{mgl}^{-1}\right)$. For secondary antibodies, we used Alexa 405-, 488-, 555-, 568-, 594- or 633-conjugated antibodies against rabbit, and mouse or rat immunoglobulin (Ig) Gs (1:250-1,000, Molecular Probes). Fluorescence imaging was conducted using scanning laser confocal microscopes IX81/FV1000 (Olympus) with a $\times 40$ objective lens (NA 1.0) and LSM-780 (Zeiss) with $\times 40$ (NA 1.1) and $\times 63$ (NA 1.4) objective lenses, and wide-field microscopes (BZ-9000, Keyence or BX-60, Olympus) with $\times 40$ and $\times 20$ objective lenses (NA 1.3, Nikon or NA 0.7, Olympus).

Image acquisition and morphometric analyses in vitro. For the accurate tracing of neurites, transfected and untransfected cells were mixed at a ratio of 1:10, grown in vitro for two days, fixed and immunostained for morphometry as follows. Pyramidal-like neurons that bore one unambiguously recognizable axon and one or more dendrites were analysed. For each neuron, the axon was discriminated from dendrites by tau-1 staining and/or an established morphological criteria (that is, relative length, diameter and position $)^{19,49}$. Axon and dendrite lengths, and branch tip numbers were measured manually using an ImageJ plugin, NeuronJ 1.1.0 (ref. 51) by an observer blinded to the identity of the samples.

Live imaging. Cerebrocortical neurons co-transfected with GFP-EB1 (ref. 52) and shSept $7 / \mathrm{mCherry}$ or mCherry by electroporation were incubated in suspension for 2 days, then plated at a density of $10^{6}$ in glass-bottom culture dishes (MatTek). At div3 (1 day after plating), the culture medium was replaced with Tyrode solution ( $129 \mathrm{mM} \mathrm{NaCl}, 5 \mathrm{mM} \mathrm{KCl}, 2 \mathrm{mM} \mathrm{CaCl}_{2}, 2 \mathrm{mM} \mathrm{MgCl}_{2}, 30 \mathrm{mM}$ D-glucose, $25 \mathrm{mM}$ Hepes, $\mathrm{pH}$ 7.4). The cells on a stage incubator at $37^{\circ} \mathrm{C}$ were imaged using an LSM780 microscope with a $\times 63$ objective lens at a rate of $1 \mathrm{~s}$ per frame for $400 \mathrm{~s}$. We randomly selected seven neurons from those that met morphological criteria, and measured the velocity of all GFP-EB1 puncta that were unambiguously tracked for $>10 \mathrm{~s}$. The movie files were reconstructed with ImageJ and the kymograph plugin was additionally used.
In situ PLA. The proximity between HDAC6 and SEPT7 in cultured neurons was assessed by in situ PLA ${ }^{30}$ using the Duolink In Situ Red kit (Olink Bioscience). The anti-SEPT7 and anti-HDAC6 antibodies were linked, respectively, to the plus and minus PLA probes, and were incubated with fixed neurons for $16 \mathrm{~h}$ at $4{ }^{\circ} \mathrm{C}$. After washing, the samples were further processed to assess whether the two antigens were close enough $(<40 \mathrm{~nm})$ to generate fluorescent signals.

Microtubule stability assay. A standard nocodazole-based microtubule stability assay ${ }^{53}$ was used with cultured mouse N-18 neural cells. Briefly, 2 days after transfection of either UI4_GFP_SIBR-SEPT7 or the cognate empty vector, cells were treated with nocodazole $(10 \mu \mathrm{M})$ for $1 \mathrm{~h}$, fixed with cold methanol and immunostained for $\alpha$-tubulin. Randomly sampled cells were line-scanned across the long axis for $\alpha$-tubulin immunofluorescence.

Immunoblotting. Samples were lysed with brief sonication in Laemmli's buffer. Polypeptides were resolved by 8 or 10\% SDS-PAGE and were transferred onto reinforced nitrocellulose or polyvinylidene difluoride membranes, which were blocked with $5 \%$ skim milk in TBST $(100 \mathrm{mM}$ Tris-HCl, $\mathrm{pH} 7.4,150 \mathrm{mM} \mathrm{NaCl}$, $0.05 \%$ Tween-20) and blotted with primary antibodies. Densitometric detection of chemiluminescence was conducted with anti-rabbit or -mouse IgG (Jackson) ECLPlus reagent (Perkin Elmer), TrueBlot anti-mouse or rabbit Ig (Rockland Immunochemicals), and an image analyser LAS-3000mini (Fuji).

Immunoprecipitation. Dispersed neurons electroporated with UI4_GFP_SIBRSEPT7 or the control plasmid were assayed at div3. HEK293T cells $\left(10^{6}\right)$ were transfected with FuGENE6 (Roche Diagnostics) to introduce the following plasmids ( $0.5 \mu \mathrm{g}$ each), pEGFP, pEGFP-SEPT7 (ref. 50), Flag (Sigma) or Flag-HDAC6 Two days later, the cells were lysed in a buffer (10 mM Tris- $\mathrm{HCl}$ at pH 7.4, $150 \mathrm{mM}$ $\mathrm{NaCl}, 1 \%$ Triton X-100), centrifuged and the supernatant was mixed with protein G or A-magnetic beads (New England Biolabs) preincubated with mouse antiFLAG M2 (Sigma), rabbit anti-HDAC6 (ref. 54), mouse anti-SIRT2 (Santa Cruz), rabbit anti-MEC-17 (LSBio), rabbit anti-ELP3 (Cell Signaling) or rabbit antiSEPT7 (ref. 10). Immune complexes on the beads were analysed by immunoblot.

In vitro binding assay. Sf9-expressed $\mathrm{His}_{6}$-tagged SEPT7/6/2 (ref. 27) was immobilized on Ni-NTA beads (Qiagen) and incubated with Sf9-expressed GSTtagged human HDAC6 (SignalChem) or GST alone in PBS for $6 \mathrm{~h}$ at $4{ }^{\circ} \mathrm{C}$. The beads were extensively washed with PBS, and subjected to SDS-PAGE and Coomassie blue staining.

Enzymatic activity measurement for HDAC6. A fluorogenic HDAC6 assay kit (BPS Bioscience) and a fluorescence microplate reader (EnSpire, Perkin Elmer) were used to measure the fluorescence intensity of a deacetylated small molecule substrate, which was incubated with each sample in $50 \mu \mathrm{l}$ at $37^{\circ} \mathrm{C}$ for $0.5 \mathrm{~h}$.

Statistical analyses. Quantitative data are shown as means \pm s.e.m. Prism 4.0 (GraphPad Software) was used for statistical analyses. $T$-test and one-way ANOVA with post hoc Tukey test were respectively applied for comparisons between two groups and three groups or more.

\section{References}

1. Mitchison, T. \& Kirschner, M. Cytoskeletal dynamics and nerve growth. Neuron 1, 761-772 (1988)

2. Conde, C. \& Caceres, A. Microtubule assembly, organization and dynamics in axons and dendrites. Nat. Rev. Neurosci. 10, 319-332 (2009).

3. Sulston, J. E. \& Horvitz, H. R. Abnormal cell lineages in mutants of the nematode Caenorhabditis elegans. Dev. Biol. 82, 41-55 (1981).

4. White, J. G., Horvitz, H. R. \& Sulston, J. E. Neurone differentiation in cell lineage mutants of Caenorhabditis elegans. Nature 297, 584-587 (1982).

5. Sakamoto, T. et al. Mass spectrometric analysis of microtubule co-sedimented proteins from rat brain. Genes Cells 13, 295-312 (2008).

6. Finger, F. P., Kopish, K. R. \& White, J. G. A role for septins in cellular and axonal migration in C. elegans. Dev. Biol. 261, 220-234 (2003).

7. Peng, X. R., Jia, Z., Zhang, Y., Ware, J. \& Trimble, W. S. The septin CDCrel-1 is dispensable for normal development and neurotransmitter release. Mol. Cell Biol. 22, 378-387 (2002).

8. Ono, R. et al. Disruption of Sept6, a fusion partner gene of $M L L$, does not affect ontogeny, leukemogenesis induced by $M L L-S E P T 6$, or phenotype induced by the loss of Sept4. Mol. Cell Biol. 25, 10965-10978 (2005).

9. Fujishima, K., Kiyonari, H., Kurisu, J., Hirano, T. \& Kengaku, M. Targeted disruption of Sept3, a heteromeric assembly partner of Sept5 and Sept7 in axons, has no effect on developing CNS neurons. J. Neurochem. 102, 77-92 (2007).

10. Ihara, M. et al. Sept4, a component of presynaptic scaffold and Lewy bodies, is required for the suppression of alpha-synuclein neurotoxicity. Neuron 53, 519-533 (2007). 
11. Tsang, C. W. et al. Superfluous role of mammalian septins 3 and 5 in neuronal development and synaptic transmission. Mol. Cell Biol. 28, 7012-7029 (2008). 12. Hall, P. A. et al. The Septins (Wiley, 2008).

13. Tooley, A. J. et al. Amoeboid T lymphocytes require the septin cytoskeleton for cortical integrity and persistent motility. Nat. Cell Biol. 11, 17-26 (2009).

14. Xie, Y. et al. The GTP-binding protein Septin 7 is critical for dendrite branching and dendritic-spine morphology. Curr. Biol. 17, 1746-1751 (2007).

15. Tada, T. et al. Role of septin cytoskeleton in spine morphogenesis and dendrite development in neurons. Curr. Biol. 17, 1752-1758 (2007).

16. $\mathrm{Hu}$, J. et al. Septin-driven coordination of actin and microtubule remodeling regulates the collateral branching of axons. Curr. Biol. 22, 1109-1115 (2012).

17. Mizuno, H., Hirano, T. \& Tagawa, Y. Evidence for activity-dependent cortical wiring: formation of interhemispheric connections in neonatal mouse visual cortex requires projection neuron activity. J. Neurosci. 27, 6760-6770 (2007).

18. Wang, C. L. et al. Activity-dependent development of callosal projections in the somatosensory cortex. J. Neurosci. 27, 11334-11342 (2007).

19. Ageta-Ishihara, N. et al. Control of cortical axon elongation by a GABA-driven $\mathrm{Ca}^{2+} /$ calmodulin-dependent protein kinase cascade. J. Neurosci. 29, 13720-13729 (2009).

20. Chung, K. H. et al. Polycistronic RNA polymerase II expression vectors for RNA interference based on BIC/miR-155. Nucleic Acids Res. 34, e53 (2006).

21. Westermann, S. \& Weber, K. Post-translational modifications regulate microtubule function. Nat. Rev. 4, 938-947 (2003).

22. Stepanova, T. et al. Visualization of microtubule growth in cultured neurons via the use of EB3-GFP (end-binding protein 3-green fluorescent protein). J. Neurosci. 23, 2655-2664 (2003).

23. Zilberman, Y. et al. Regulation of microtubule dynamics by inhibition of the tubulin deacetylase HDAC6. J. Cell Sci. 122, 3531-3541 (2009).

24. Hubbert, C. et al. HDAC6 is a microtubule-associated deacetylase. Nature 417, 455-458 (2002)

25. North, B. J., Marshall, B. L., Borra, M. T., Denu, J. M. \& Verdin, E. The human Sir2 ortholog, SIRT2, is an $\mathrm{NAD}^{+}{ }^{-}$dependent tubulin deacetylase. Mol. Cell 11, 437-444 (2003)

26. Southwood, C. M., Peppi, M., Dryden, S., Tainsky, M. A. \& Gow, A. Microtubule deacetylases, SirT2 and HDAC6, in the nervous system. Neurochem. Res. 32, 187-195 (2007).

27. Kinoshita, M., Field, C. M., Coughlin, M. L., Straight, A. F. \& Mitchison, T. J. Self- and actin-templated assembly of mammalian septins. Dev. Cell 3, 791-802 (2002).

28. Akella, J. S. et al. MEC-17 is an alpha-tubulin acetyltransferase. Nature 467, 218-222 (2010)

29. Creppe, C. et al. Elongator controls the migration and differentiation of cortical neurons through acetylation of alpha-tubulin. Cell 136, 551-564 (2009).

30. Soderberg, O. et al. Direct observation of individual endogenous protein complexes in situ by proximity ligation. Nat. Methods 3, 995-1000 (2006).

31. Haggarty, S. J., Koeller, K. M., Wong, J. C., Grozinger, C. M. \& Schreiber, S. L. Domain-selective small-molecule inhibitor of histone deacetylase 6 (HDAC6)mediated tubulin deacetylation. Proc. Natl Acad. Sci. USA 100, 4389-4394 (2003).

32. Caudron, F. \& Barral, Y. Septins and the lateral compartmentalization of eukaryotic membranes. Dev. Cell 16, 493-506 (2009).

33. Yang, Y. M. et al. Septins regulate developmental switching from microdomain to nanodomain coupling of $\mathrm{Ca}^{2+}$ influx to neurotransmitter release at a central synapse. Neuron 67, 100-115 (2010).

34. Sharma, S. et al. An siRNA screen for NFAT activation identifies septins as coordinators of store-operated $\mathrm{Ca}^{2}+$ entry. Nature 499, 238-242 (2013).

35. Spiliotis, E. T., Hunt, S. J., Hu, Q., Kinoshita, M. \& Nelson, W. J. Epithelial polarity requires septin coupling of vesicle transport to polyglutamylated microtubules. J. Cell Biol. 180, 295-303 (2008)

36. Bowen, J. R., Hwang, D., Bai, X., Roy, D. \& Spiliotis, E. T. Septin GTPases spatially guide microtubule organization and plus end dynamics in polarizing epithelia. J. Cell Biol. 194, 187-197 (2011).

37. Kremer, B. E., Haystead, T. \& Macara, I. G. Mammalian septins regulate microtubule stability through interaction with the microtubule-binding protein MAP4. Mol. Biol. Cell 16, 4648-4659 (2005)

38. Valenzuela-Fernandez, A., Cabrero, J. R., Serrador, J. M. \& Sanchez-Madrid, F. HDAC6: a key regulator of cytoskeleton, cell migration and cell-cell interactions. Trends. Cell Biol. 18, 291-297 (2008).

39. Pennington, K. et al. Prominent synaptic and metabolic abnormalities revealed by proteomic analysis of the dorsolateral prefrontal cortex in schizophrenia and bipolar disorder. Mol. Psychiatry 13, 1102-1117 (2008).

40. Zhang, Y. et al. Mice lacking histone deacetylase 6 have hyperacetylated tubulin but are viable and develop normally. Mol. Cell Biol. 28, 1688-1701 (2008).

41. Espallergues, J. et al. HDAC6 regulates glucocorticoid receptor signaling in serotonin pathways with critical impact on stress resilience. J. Neurosci. 32, 4400-4416 (2012)

42. Fukada, M. et al. Loss of deacetylation activity of Hdac6 affects emotional behavior in mice. PLoS One 7, e30924 (2012).
43. d'Ydewalle, C. et al. HDAC6 inhibitors reverse axonal loss in a mouse model of mutant HSPB1-induced Charcot-Marie-Tooth disease. Nat. Med. 17, 968-974 (2011).

44. Taes, I. et al. Hdac6 deletion delays disease progression in the $S O D 1^{\mathrm{G} 93 \mathrm{~A}}$ mouse model of ALS. Hum. Mol. Genet. 22, 1783-1790 (2013).

45. Kim, A. H. et al. A centrosomal Cdc20-APC pathway controls dendrite morphogenesis in postmitotic neurons. Cell 136, 322-336 (2009).

46. Tapia, M., Wandosell, F. \& Garrido, J. J. Impaired function of HDAC6 slows down axonal growth and interferes with axon initial segment development. PLoS One 5, e12908 (2010).

47. Du, G. et al. Drosophila histone deacetylase 6 protects dopaminergic neurons against $\alpha$-synuclein toxicity by promoting inclusion formation. Mol. Biol. Cell 21, 2128-2137 (2004).

48. Shaner, N. C. et al. Improved monomeric red, orange and yellow fluorescent proteins derived from Discosoma sp. red fluorescent protein. Nat. Biotechnol. 22, 1567-1572 (2004).

49. Takemoto-Kimura, S. et al. Regulation of dendritogenesis via a lipid-raftassociated $\mathrm{Ca}^{2+}$ /calmodulin-dependent protein kinase CLICK-III/ CaMKIgamma. Neuron 54, 755-770 (2007).

50. Hagiwara, A. et al. Submembranous septins as relatively stable components of actin-based membrane skeleton. Cytoskeleton (Hoboken) 68, 512-525 (2011).

51. Meijering, E. et al. Design and validation of a tool for neurite tracing and analysis in fluorescence microscopy images. Cytometry A. 58, 167-176 (2004)

52. Mimori-Kiyosue, Y., Shiina, N. \& Tsukita, S. The dynamic behavior of the APC-binding protein EB1 on the distal ends of microtubules. Curr. Biol. 10, 865-868 (2000).

53. Nguyen, H. L. et al. Overexpression of full- or partial-length MAP4 stabilizes microtubules and alters cell growth. J. Cell Sci. 110(Pt 2): 281-294 (1997).

54. Gao, Y. S. et al. Histone deacetylase 6 regulates growth factor-induced actin remodeling and endocytosis. Mol. Cell Biol. 27, 8637-8647 (2007).

55. Matsuyama, A. et al. In vivo destabilization of dynamic microtubules by HDAC6-mediated deacetylation. EMBO J. 21, 6820-6831 (2002).

\section{Acknowledgements}

We thank M. Masaoka and Y. Enomoto (Nagoya University), respectively for the technical help in in utero electroporation and FACS analysis, T-P. Yao and Y-S. Gao (Duke University) for HDAC6 antibody, E.T. Spiliotis (Drexel University) for polyglutamylated tubulin antibody, Y. Kiyosue (RIKEN CDB) for GFP-EB1 plasmid, M. Yoshida (RIKEN) for HDAC6-HA plasmid, and R.Y. Tsien (University of California, San Diego) for mCherry cDNA. A part of the microscopic analysis was done at Nagoya University Advanced Live Imaging Center funded by Japan Advanced Plant Science Network. This work was supported in part by Grant-in-Aid for Scientific Research (B) (to M.K.), Grant-in-Aid for Young Investigator (B) (to N.A.-I.), Grants-in-Aid for Scientific Research on Innovative Areas 'Synapse Neurocircuit Pathology' and 'Micro Psychopathology' (to M.K.), 'Cross-talk between moving cells and microenvironment as a basis of emerging order in multicellular system' (to T.M.), Technical Supports for Fluorescent Probes and Imaging and for Antibody Production from Comprehensive Brain Science Network (to N.A.-I., M.W., H.B., M.K.), each from the Ministry of Education, Science, Sports and Culture of Japan, and by the research grants from Takeda Science Foundation, Astellas Foundation, Suzuken Memorial Foundation, Narishige Neuroscience Research Foundation (to N.A.-I.), and NIH grant P50A086355 (to R.M.)

\section{Author contributions}

N.A.-I. designed and performed most of the experiments and wrote the manuscript. T.M. supervised in utero electroporation, FACS and perinatal sample preparations. C.O. assisted biochemical experiments. Y.S., Y.H. and T.H. supported microscopy and live image data processing. R.M. and M.W. provided reagents. H.B. and M.K. designed and supervised the study and wrote the manuscript. The authors declare no competing financial interests.

\section{Additional information}

Supplementary Information accompanies this paper at http://www.nature.com/ naturecommunications

Competing financial interests: The authors declare no competing financial interests.

Reprints and permission information is available online at http://npg.nature.com/ reprintsandpermissions/

How to cite this article: Ageta-Ishihara, N. et al. Septins promote dendrite and axon development by negatively regulating microtubule stability via HDAC6-mediated deacetylation. Nat. Commun. 4:2532 doi: 10.1038/ncomms3532 (2013).

This work is licensed under a Creative Commons AttributionNonCommercial-NoDerivs 3.0 Unported License. To view a copy of this license, visit http://creativecommons.org/licenses/by-nc-nd/3.0/ 